\title{
Effects of neutrino mass hierarchies on dynamical dark energy models
}

\author{
Weiqiang Yang, ${ }^{1, *}$ Rafael C. Nunes, ${ }^{2, \dagger}$ Supriya Pan, ${ }^{3, \$}$ and David F. Mota ${ }^{4, \S}$ \\ ${ }^{1}$ Department of Physics, Liaoning Normal University, Dalian 116029, People's Republic of China \\ ${ }^{2}$ Departamento de Física, Universidade Federal de Juiz de Fora, Juiz de Fora, \\ Minas Gerais 36036-330, Brazil \\ ${ }^{3}$ Department of Physical Sciences, Indian Institute of Science Education and Research-Kolkata, \\ Mohanpur, West Bengal 741246 , India \\ ${ }^{4}$ Institute of Theoretical Astrophysics, University of Oslo, 0315 Oslo, Norway \\ (Received 15 February 2017; revised manuscript received 10 April 2017; published 31 May 2017)
}

\begin{abstract}
We investigate how three different possibilities of neutrino mass hierarchies, namely normal, inverted, and degenerate, can affect the observational constraints on three well-known dynamical dark energy models, namely the Chevallier-Polarski-Linder, logarithmic, and the Jassal-Bagla-Padmanabhan parametrizations. In order to impose the observational constraints on the models, we performed a robust analysis using Planck 2015 temperature and polarization data, supernovae type Ia from the joint light curve analysis, baryon acoustic oscillation distance measurements, redshift space distortion characterized by $f(z) \sigma_{8}(z)$ data, weak gravitational lensing data from the Canada-France-Hawaii Telescope Lensing Survey, and cosmic chronometer data plus the local value of the Hubble parameter. We find that different neutrino mass hierarchies return similar fits on almost all model parameters and mildly change the dynamical dark energy properties.
\end{abstract}

DOI: 10.1103/PhysRevD.95.103522

\section{INTRODUCTION}

The hot big bang model predicts the existence of a cosmic neutrino background which has not been directly detected, but has indirectly been established by using cosmic microwave background (CMB) observations, as well as estimations from the primordial abundances of light elements. On the other hand, the phenomena of neutrino oscillation from several experiments have shown that neutrinos have very small but nonzero masses; see [1] for review. For instance, measures from solar neutrino analysis supplemented by the KamLAND estimate $\Delta m_{21}^{2} \equiv m_{2}^{2}-m_{1}^{2} \simeq 8 \times 10^{-5} \mathrm{eV}^{2}$ [2], and experiments of atmospheric neutrino oscillation by Super-Kamiokande show $\left|\Delta m_{31}^{2}\right| \equiv\left|m_{3}^{2}-m_{1}^{2}\right| \simeq 3 \times 10^{-3} \mathrm{eV}^{2}$ [3]. Unfortunately, the current oscillation experiments do not give much information about the absolute scale of neutrino masses, since the measures of $\Delta m_{21}^{2}>0$ and $\left|\Delta m_{31}^{2}\right|$ lead us to two different possible scenarios of mass hierarchies, which are known as the normal hierarchy $(\mathrm{NH})$ and the inverted hierarchy (IH), characterized respectively by the positive and negative sign of the quantity $\left|\Delta m_{31}^{2}\right|$. Within the $\mathrm{NH}$ scenario, one eigenstate is much heavier and the lower bound is constrained to $\sum m_{\nu}=0.06 \mathrm{eV}$ [4] [at $95 \%$ confidence level (C.L.)]. In the IH scenario, the two heaviest neutrinos are nearly degenerate and the lower bound is $\sum m_{\nu}=0.10 \mathrm{eV}$ [4] (95\% C.L.). On the other hand, from the point of view of

\footnotetext{
*d11102004@mail.dlut.edu.cn

rcnunes@fisica.ufjf.br

span@iiserkol.ac.in

§mota@astro.uio.no
}

the cosmological restrictions on the neutrino mass bound, one may consider one other phenomenological hierarchy, the so-called degenerate hierarchy (DH), where the masses of the neutrinos are much larger than the differences between them; hence all three active neutrinos are considered to share the same mass. However, $\mathrm{NH}$ and $\mathrm{IH}$ are two real physical possibilities from the particle physics experiments. The possibility that all neutrino masses are virtually the same is not completely excluded by future measurements of absolute mass [5]. Since the cosmological data do not have sufficient sensitivity to measure individual masses, it is reasonable to consider the total neutrino mass as $\sum m_{\nu} \simeq$ $3 m_{1} \mathrm{eV}$ (considering three active neutrinos), and a lower bound as $\sum m_{\nu}=0 \mathrm{eV}$ for the $\mathrm{DH}$ scheme. For a general discussion about neutrino mass hierarchies we refer to [6], and the works [7-12] for cosmological constraints on neutrino mass hierarchies.

Massive neutrinos play an important role in the dynamics of the Universe affecting important cosmological information sources, for instance, the formation of the large scale structure, big bang nucleosynthesis, and CMB anisotropies (see $[13,14]$ for review). The Planck Collaboration [15] within the base $\Lambda \mathrm{CDM}+\sum m_{\nu}$ model has constrained the total neutrino mass to $\sum m_{\nu}<0.194 \mathrm{eV}$ (from CMB alone), and the effective number of neutrino species, $N_{\text {eff }}=$ $3.04 \pm 0.33$ at $2 \sigma$ C.L. An extended $\Lambda \mathrm{CDM}+c_{\text {eff }}+c_{\text {vis }}+$ $\sum m_{\nu}$ model has provided $\sum m_{\nu}<0.88 \mathrm{eV}$ at $95 \%$ C.L. (from CMB alone) [16]. A forecast on neutrinos from the CORE space mission was reported in [17]. Additionally, the cosmological consequences of the massive neutrinos have been investigated in the context of $f(R)$ gravity 
[18,19], holographic dark energy [20,21], scalar field models [22-24], and coupled dark energy [25-27]. Furthermore, the presence of massive neutrinos can also reconcile the current tension on the local and global Hubble constant measures [28-30]. Also, the neutrino properties have been considered in the estimation of the inflationary parameters [31-33].

The aim of the present work is to investigate how different neutrino mass hierarchical (or the ordering of the neutrino masses) scenarios can correlate with the other cosmological parameters in the presence of dynamical dark energy (DE) models. Since there are dozens of different dark energy models [34-59], in this work we consider three general parametric models for DE, namely the Chevallier-PolarskiLinder (CPL) parametrization, the logarithmic model, and the Jassal-Bagla-Padmanabhan (JBP) parametric model. The dynamical models of DE have been recently constrained from other observational perspectives [60-63]. The paper is organized as follows. In the next section we introduce the dynamical dark energy models and their perturbation equations. Section III describes the observational data that we employ in our analysis. In Sec. IV we discuss the results of our analysis for all models. Finally, we close our work in Sec. V with a short summary of the whole work.

\section{DYNAMICAL DARK ENERGY}

Let us consider a spatially flat Friedmann-LemaîtreRobertson-Walker (FLRW) universe which is filled with photons $(\gamma)$, neutrinos $(\nu)$, baryons $(b)$, dark matter $(d m)$, and dark energy (de) fluids. The Friedmann's equations in such a universe can be written as

$$
\begin{aligned}
H^{2} & =\frac{8 \pi G}{3}\left(\rho_{\gamma}+\rho_{\nu}+\rho_{b}+\rho_{d m}+\rho_{d e}\right), \\
2 \dot{H}+3 H^{2} & =-8 \pi G\left(p_{\gamma}+p_{\nu}+p_{b}+p_{d m}+p_{d e}\right)
\end{aligned}
$$

where $\rho_{i}$ 's and $p_{i}$ 's $(i=\gamma, \nu, b, d m, d e)$ are respectively the energy density and the pressure of the $i$ th component of the fluid. Moreover, we also consider that the fluid components do not interact with each other. In other words, each component is conserved separately. That means the balance equation reads

$$
\dot{\rho}_{i}+3 H\left(p_{i}+\rho_{i}\right)=0 .
$$

Now, if the dark energy is of a dynamical nature, then its evolution is governed by the following equation,

$$
\rho_{d e}=\rho_{d e 0}\left(\frac{a}{a_{0}}\right)^{-3} \exp \left(-3 \int_{a_{0}}^{a} \frac{w_{d e}}{a^{\prime}} d a^{\prime}\right),
$$

where $\rho_{d e, 0}$ is the present value of $\rho_{d e}, a_{0}$ is the present value of the scale factor and $1+z=a_{0} / a$. In the rest of our analysis we shall consider $a_{0}=1$. Now, from Eq. (4) it is evident that if $w_{d e}$ is specified, one can understand the possible evolution of the DE in the FLRW universe. In our study we mainly concentrate on three dynamical DE models, namely (i) the CPL parametrization $[64,65]$, (ii) the logarithmic parametrization [66], and (iii) the JBP parametrization [67]. In what follows, we specify the basic equations that describe the evolution and dynamics of the dark energy components under such parametrizations.

\section{A. Chevallier-Polarski-Linder model}

A simple parameterization was introduced in $[64,65]$ to investigate the possible dynamical aspects of DE,

$$
w_{d e}=w_{0}+w_{1}(1-a),
$$

where $w_{0}, w_{1}$ are the free parameters of the model and physically $w_{0}=w_{d e}(z=0)$; i.e., it is the present value of the DE density and $w_{1}=d w /\left.d z\right|_{(z=0)}$. The same notation is maintained for the next two models. Now, for this parametrization the DE evolution is described by

$$
\rho_{d e}=\rho_{d e, 0} a^{-3\left(1+w_{0}+w_{1}\right)} \exp \left[-3 w_{1}(1-a)\right],
$$

where $\rho_{d e, 0}$ is the current value of DE density.

\section{B. The logarithmic model}

Let us recall another parametrization introduced by Efstathiou [66] in which the equation of state (EoS) is characterized by a logarithmic law

$$
w_{d e}=w_{0}-w_{1} \ln a,
$$

and the DE for this EoS evolves as

$$
\rho_{d e}=\rho_{d e, 0} a^{-3\left(1+w_{0}\right)} \exp \left[\frac{3 w_{1}}{2}(\ln a)^{2}\right],
$$

and commonly this parametrization is known as the logarithmic parametrization.

\section{Jassal-Bagla-Padmanabhan model}

Let us introduce another DE parametrization

$$
w_{d e}=w_{0}+w_{1} a(1-a),
$$

where the DE evolves as

$$
\rho_{d e}=\rho_{d e, 0} a^{-3\left(1+w_{0}\right)} \exp \left(\frac{3 w_{1}}{2}(a-1)^{2}\right) .
$$

This parametrization is known as the JBP parametrization.

\section{Linear perturbations}

Let us now review the linear perturbation equations. The most general scalar mode perturbation is defined by the following metric [68-70]:

$$
\begin{aligned}
d s^{2}= & -(1+2 \phi) d t^{2}+2 a \partial_{i} B d t d x \\
& +a^{2}\left[(1-2 \psi) \delta_{i j}+2 \partial_{i} \partial_{j} E\right] d x^{i} d x^{j} .
\end{aligned}
$$


Here, we follow [69], and let us adopt the synchronous gauge, i.e. $\phi=B=0, \psi=\eta$, and $k^{2} E=-h / 2-3 \eta$. The energy and momentum conservation equations for the $i$ th component of the fluid in the synchronous gauge are given by

$$
\begin{array}{r}
\delta_{i}^{\prime}=-\left(1+w_{i}\right)\left(\theta_{i}+\frac{h^{\prime}}{2}\right)-3 \mathcal{H}\left(\frac{\delta P_{i}}{\delta \rho_{i}}-w_{i}\right) \delta_{i}, \\
\theta_{i}^{\prime}=-\mathcal{H}\left(1-3 w_{i}\right) \theta_{i}-\frac{w_{i}^{\prime}}{1+w_{i}} \theta_{i}+\frac{\delta P_{i} / \delta \rho_{i}}{1+w_{i}} k^{2} \delta_{i}-k^{2} \sigma
\end{array}
$$

where the prime denotes the derivative with respect to conformal time; $\mathcal{H}$ is the conformal Hubble function; the quantities $\sigma, \delta_{i}, \theta_{i}$, are respectively the shear, density perturbation, and velocity perturbation. The DE perturbations can be written as

$$
\begin{aligned}
& \delta_{d e}^{\prime}=-\left(1+w_{d e}\right)\left(\theta_{d e}+\frac{h^{\prime}}{2}\right)-3 \mathcal{H} w_{d e}^{\prime} \frac{\theta_{d e}}{k^{2}} \\
&-3 \mathcal{H}\left(c_{s}^{2}-w_{d e}\right)\left[\delta_{d e}+3 \mathcal{H}\left(1+w_{d e}\right) \frac{\theta_{d e}}{k^{2}}\right], \\
& \theta_{d e}^{\prime}=-\mathcal{H}\left(1-3 c_{s}^{2}\right) \theta_{d e}+\frac{c_{s}^{2}}{1+w_{d e}} k^{2} \delta_{d e}
\end{aligned}
$$

where for simplicity we considered $\sigma=0$. Here, $c_{s}^{2}$ is the physical sound speed in the rest frame. In order to avoid the unphysical sound speed we assume $c_{s}^{2}=1$. Since the baryons, dark matter, photons and massive neutrinos are conserved independently, thus, the perturbation equations for each component follow the standard evolution described in [68-70].

\section{DATA SET AND METHODOLOGY}

In what follows, we briefly describe the observational data sets used in this work.

(i) Cosmic microwave background data $(C M B): \mathrm{We}$ use CMB data from the Planck 2015 measurements [71,72], where we combine the likelihoods $C_{l}^{T T}, C_{l}^{E E}$, $C_{l}^{T E}$ in addition to low- $l$ polarization $C_{l}^{T E}+C_{l}^{E E}+$ $C_{l}^{B B}$, which notationally is same as "Planck TT, EE, TE + lowTEB" of Ref. [15].

(ii) Supernovae type Ia (SNIa): We take the latest joint light curves (JLA) sample [73] containing 740 SNIa in the redshift range $z \in[0.01,1.30]$.

(iii) Baryon acoustic oscillation (BAO) distance measurements: For the BAO data we use the estimated ratio $r_{s} / D_{V}$ as a "standard ruler" in which $r_{s}$ is the comoving sound horizon at the baryon drag epoch and $D_{V}$ is the effective distance determined by the angular diameter distance $D_{A}$ and Hubble parameter $H$ as $D_{V}(z)=\left[(1+z)^{2} D_{A}(a)^{2} \frac{z}{H(z)}\right]^{1 / 3}$. We consider three different measurements: $r_{s}\left(z_{d}\right) / D_{V}(z=0.106)=$ $0.336 \pm 0.015$ from the 6-degree Field Galaxy Redshift Survey (6dFGRS) data [74], $r_{s}\left(z_{d}\right) / D_{V}(z=$ $0.35)=0.1126 \pm 0.0022$ from the Sloan Digital

TABLE I. The observational constraints on the free parameters and the derived parameters $\left(\sigma_{8}, H_{0}, \Omega_{m 0}, \Omega_{\nu} h^{2}\right)$ of the CPL

\begin{tabular}{|c|c|c|c|}
\hline \multirow[b]{2}{*}{ Parameters } & \multicolumn{3}{|c|}{ CPL model } \\
\hline & Normal hierarchy & Inverted hierarchy & Degenerate hierarchy \\
\hline$\Omega_{d m} h^{2}$ & $0.1181_{-0.0012-0.0023}^{+0.0012+0.0024}$ & $0.1182_{-0.0012-0.0025}^{+0.0012+0.0024}$ & $0.1182_{-0.0012-0.0024}^{+0.0012+0.0025}$ \\
\hline$\Omega_{b} h^{2}$ & $0.0223_{-0.0001-0.0003}^{+0.0001+0.003}$ & $0.0223_{-0.0001-0.0003}^{+0.00001+0.0003}$ & $0.0223_{-0.0001-0.0003}^{+0.0001+0.003}$ \\
\hline $100 \theta_{M C}$ & $1.0408_{-0.0003-0.0006}^{+0.0003+0.006}$ & $1.0408_{-0.0003-0.0006}^{+0.0003+0.0006}$ & $1.0408_{-0.0003-0.0006}^{+0.0003+0.006}$ \\
\hline$\tau_{\text {reio }}$ & $0.0680_{-0.0168-0.0348}^{+0.0170+0.0332}$ & $0.0706_{-0.0171-0.0350}^{+0.0188+0.0334}$ & $0.0680_{-0.0178-0.0351}^{+0.0181+0.0354}$ \\
\hline$n_{s}$ & $0.9682_{-0.0043-0.0083}^{+0.0043+0.0055}$ & $0.9678_{-0.0046-0.0082}^{+0.0042+0.0087}$ & $0.9677_{-0.0042-0.0083}^{+0.0042+0.0084}$ \\
\hline $\ln \left(10^{10} A_{s}\right)$ & $3.0655_{-0.0325-0.0670}^{+0.0328+0.0650}$ & $3.0706_{-0.0341-0.0683}^{+0.0342+0.0649}$ & $3.0658_{-0.0345-0.0682}^{+0.0350+0.0685}$ \\
\hline$w_{0}$ & $-0.9414_{-0.1172-0.2016}^{+0.1007+0.2062}$ & $-0.9323_{-0.1298-0.2056}^{+0.0957+0.2187}$ & $-0.9164_{-0.1135-0.2080}^{+0.1001+0.2127}$ \\
\hline$w_{1}$ & $-0.4303_{-0.3401-0.8888}^{+0.5402+0.8084}$ & $-0.5230_{-0.3210-0.9270}^{+0.5463+8.823}$ & $-0.5564_{-0.3626-1.9445}^{+0.5524+0.9207}$ \\
\hline$\Sigma m_{\nu}$ & $<0.3538$ (95\% C.L.) & $<0.3686$ (95\% C.L.) & $<0.4272(95 \%$ C.L. $)$ \\
\hline$\sigma_{8}$ & $0.8082_{-0.0152-0.0390}^{+0.0211+0.0351}$ & $0.8047_{-0.0160-0.0351}^{+0.0178+0.0350}$ & $0.8068_{-0.0166-0.0431}^{+0.0236+0.0388}$ \\
\hline$H_{0}$ & $68.5265_{-0.9353-1.6824}^{+0.8406+1.7887}$ & $68.4954_{-0.8758-1.7443}^{+0.8675+1.7329}$ & $68.4419_{-0.8708-1.6711}^{+0.8776+1.7318}$ \\
\hline$\Omega_{\nu} h^{2}$ & $0.0017_{-0.0012-0.0014}^{+0.0004+0.0024}$ & $0.0022_{-0.0011-0.0014}^{+0.0004+0.0019}$ & $0.0019_{-0.0019-0.0022}^{+0.0007+0.0029}$ \\
\hline$\Omega_{m 0}$ & $0.3029_{-0.0087-0.0169}^{+0.0086+0.0178}$ & $0.3042_{-0.0093-0.0162}^{+0.0084+0.0175}$ & $0.3042_{-0.0090-0.0172}^{+0.0089+0.0183}$ \\
\hline$\chi_{\min }^{2}$ & 13720.722 & 13722.104 & 13720.610 \\
\hline
\end{tabular}
parametrization for three different neutrino mass hierarchies, namely normal hierarchy, inverted hierarchy and the degenerate hierarchy, using the observational data combinations $\mathrm{CMB}+\mathrm{SNIa}+\mathrm{BAO}+\mathrm{RSD}+\mathrm{WL}+\mathrm{CC}+H_{0}$. Mean values of the parameters are displayed at $1 \sigma(68 \%)$ and $2 \sigma(95 \%)$ errors. The parameter $H_{0}$ is in the units of $\mathrm{km} / \mathrm{s} / \mathrm{Mpc}, \sum m_{\nu}$ is in the units of eV with a $2 \sigma$ upper bound, and $\Omega_{m 0}=\Omega_{d m 0}+\Omega_{b 0}$. 
TABLE II. The observational constraints on the free parameters and the derived parameters $\left(\sigma_{8}, H_{0}, \Omega_{m 0}, \Omega_{\nu} h^{2}\right)$ of the logarithmic parametrization for three different neutrino mass hierarchies, namely the normal hierarchy, inverted hierarchy and the degenerate hierarchy, using the observational data combinations $\mathrm{CMB}+\mathrm{SNIa}+\mathrm{BAO}+\mathrm{RSD}+\mathrm{WL}+\mathrm{CC}+H_{0}$. Mean values of the parameters are displayed at $1 \sigma(68 \%)$ and $2 \sigma(95 \%)$ errors. The parameter $H_{0}$ is in the units of $\mathrm{km} / \mathrm{s} / \mathrm{Mpc}, \sum m_{\nu}$ is in the units of eV with a $2 \sigma$ upper bound, and $\Omega_{m 0}=\Omega_{d m 0}+\Omega_{b 0}$.

\begin{tabular}{|c|c|c|c|}
\hline \multirow[b]{2}{*}{ Parameters } & \multicolumn{3}{|c|}{ Logarithmic model } \\
\hline & Normal hierarchy & Inverted hierarchy & Degenerate hierarchy \\
\hline$\Omega_{d m} h^{2}$ & $0.1184_{-0.0012-0.0025}^{+0.0012+0.0023}$ & $0.1184_{-0.0012-0.0025}^{+0.0012+0.0024}$ & $0.1184_{-0.0012-0.0025}^{+0.0012+0.0025}$ \\
\hline$\Omega_{b} h^{2}$ & $0.0223_{-0.0001-0.0003}^{+0.0001+0.0003}$ & $0.0223_{-0.0002-0.0003}^{+0.0002+0.0003}$ & $0.0223_{-0.0001-0.0003}^{+0.0002+0.0003}$ \\
\hline $100 \theta_{M C}$ & $1.0408_{-0.0003-0.0007}^{+0.0003+0.0006}$ & $1.0408_{-0.0003-0.0006}^{+0.0003+0.0006}$ & $1.0408_{-0.0003-0.0006}^{+0.0003+0.0006}$ \\
\hline$\tau_{\text {reio }}$ & $0.0685_{-0.0169-0.0332}^{+0.0171+0.0338}$ & $0.0683_{-0.0178-0.0351}^{+0.0181+0.0352}$ & $0.0669_{-0.0178-0.0355}^{+0.0181+0.0355}$ \\
\hline$n_{s}$ & $0.9676_{-0.0044-0.0086}^{+0.0045+0.0087}$ & $0.9674_{-0.0044-0.0085}^{+0.0044+0.0085}$ & $0.9674_{-0.0042-0.0086}^{+0.0043+0.0085}$ \\
\hline $\ln \left(10^{10} A_{s}\right)$ & $3.0670_{-0.0332-0.0643}^{+0.0334+0.0650}$ & $3.0666_{-0.0339-0.0669}^{+0.0353+0.0689}$ & $3.0642_{-0.0343-0.0685}^{+0.0350+0.0678}$ \\
\hline$w_{0}$ & $-0.9074_{-0.0962-0.1628}^{+0.0728+0.1688}$ & $-0.9147_{-0.1035-0.1707}^{+0.0763+0.1789}$ & $-0.9143_{-0.0958-0.1563}^{+0.0683+0.1765}$ \\
\hline$w_{1}$ & $-0.4727_{-0.1479-0.6136}^{+0.4055+0.4727}$ & $-0.4904_{-0.1425-0.6493}^{+0.4404+0.4904}$ & $-0.4358_{-0.0986-0.6459}^{+0.4358+0.4358}$ \\
\hline$\Sigma m_{\nu}$ & $<0.412$ (95\% C.L.) & $<0.428$ (95\% C.L.) & $<0.425$ (95\% C.L.) \\
\hline$\sigma_{8}$ & $0.8072_{-0.0149-0.0406}^{+0.0208+0.0378}$ & $0.8036_{-0.0154-0.0395}^{+0.0207+0.0372}$ & $0.8092_{-0.0176-0.0455}^{+0.0242+0.0403}$ \\
\hline$H_{0}$ & $68.3616_{-0.8735-1.6450}^{+0.8455+1.6910}$ & $68.4264_{-0.8408-1.6902}^{+0.8587+1.6949}$ & $68.4151_{-0.8734-1.6691}^{+0.8364+1.7241}$ \\
\hline$\Omega_{\nu} h^{2}$ & $0.0020_{-0.0014-0.0018-0.0013}^{+0.0004+0.0026}$ & $0.0023_{-0.0013-0.0016}^{+0.0004+0.0024}$ & $0.0018_{-0.0018-0.0021}^{+0.0007+0.0030}$ \\
\hline$\Omega_{m 0}$ & $0.3054_{-0.0091-0.0159}^{+0.0083+0.0178}$ & $0.3055_{-0.0094-0.0172}^{+0.0084+0.0176}$ & $0.3045_{-0.0097-0.0163}^{+0.0081+0.0181}$ \\
\hline$\chi_{\min }^{2}$ & 13723.006 & 13721.892 & 13722.96 \\
\hline
\end{tabular}

TABLE III. The observational constraints on the free parameters and the derived parameters $\left(\sigma_{8}, H_{0}, \Omega_{m 0}, \Omega_{\nu} h^{2}\right)$ of the JBP parametrization for three different neutrino mass hierarchies, namely the normal hierarchy, inverted hierarchy and the degenerate hierarchy, using the observational data combinations $\mathrm{CMB}+\mathrm{SNIa}+\mathrm{BAO}+\mathrm{RSD}+\mathrm{WL}+\mathrm{CC}+H_{0}$. Mean values of the parameters are displayed at $1 \sigma(68 \%)$ and $2 \sigma(95 \%)$ errors. The parameter $H_{0}$ is in the units of $\mathrm{km} / \mathrm{s} / \mathrm{Mpc}, \sum m_{\nu}$ is in the units of eV with a $2 \sigma$ upper bound, and $\Omega_{m 0}=\Omega_{d m 0}+\Omega_{b 0}$.

\begin{tabular}{|c|c|c|c|}
\hline \multirow[b]{2}{*}{ Parameters } & \multicolumn{3}{|c|}{ JBP model } \\
\hline & Normal hierarchy & Inverted hierarchy & Degenerate hierarchy \\
\hline$\Omega_{d m} h^{2}$ & $0.1179_{-0.0013-0.0025}^{+0.0012+0.0024}$ & $0.1179_{-0.0012-0.0024}^{+0.0012+0.0023}$ & $0.1180_{-0.0012-0.0025}^{+0.0012+0.0024}$ \\
\hline$\Omega_{b} h^{2}$ & $0.0223_{-0.0001-0.0003}^{+0.0002+0.0003}$ & $0.0223_{-0.0001-0.0003}^{+0.0001+0.0003}$ & $0.0223_{-0.0001-0.0003}^{+0.0001+0.00029}$ \\
\hline $100 \theta_{M C}$ & $1.0409_{-0.0003-0.0006}^{+0.0003+0.0006}$ & $1.0408_{-0.0003-0.0006}^{+0.0003+0.0006}$ & $1.0409_{-0.0003-0.0006}^{+0.0003+0.0006}$ \\
\hline$\tau_{\text {reio }}$ & $0.0703_{-0.0182-0.0341}^{+0.0171+0.0346}$ & $0.0708_{-0.0170-0.0334}^{+0.0171+0.0337}$ & $0.0680_{-0.0177-0.0340}^{+0.0178+0.0348}$ \\
\hline$n_{s}$ & $0.9689_{-0.0047-0.0085}^{+0.0041+0.0088}$ & $0.9686_{-0.0041-0.0080}^{+0.0041+0.0082}$ & $0.9686_{-0.0041-0.0086}^{+0.0042+0.0084}$ \\
\hline $\ln \left(10^{10} A_{s}\right)$ & $3.0701_{-0.0331-0.0650}^{+0.0332+0.0665}$ & $3.0706_{-0.0328-0.0650}^{+0.0328+0.0655}$ & $3.0658_{-0.0343-0.0658}^{+0.0342+0.0673}$ \\
\hline$w_{0}$ & $-0.9628_{-0.1846-0.2900}^{+0.1454+0.3222}$ & $-0.9329_{-0.1572-0.2767}^{+0.1443+0.2802}$ & $-0.9658_{-0.1344-0.2372}^{+0.1163+0.2602}$ \\
\hline$w_{1}$ & $-0.5080_{-0.8641-2.0100}^{+1.1835+1.6937}$ & $-0.7903_{-0.8594-1.8009}^{+0.9842+1.6452}$ & $-0.3964_{-0.6569-1.6432}^{+0.8292+1.4567}$ \\
\hline$\sum m_{\nu}$ & $<0.294$ (95\% C.L.) & $<0.348$ (95\% C.L.) & $<0.253$ (95\% C.L.) \\
\hline$\sigma_{8}$ & $0.8107_{-0.0153-0.0325}^{+0.0166+0.0303}$ & $0.8028_{-0.0155-0.0328}^{+0.0179+0.0307}$ & $0.8153_{-0.0164-0.0345}^{+0.0166+0.0321}$ \\
\hline$H_{0}$ & $68.6228_{-1.0041-1.7978}^{+0.9276+1.9123}$ & $68.5100_{-0.9235-1.8080}^{+0.9122+1.8247}$ & $68.5621_{-0.8739-1.6835}^{+0.8695+1.7740}$ \\
\hline$\Omega_{\nu} h^{2}$ & $0.0015_{-0.0010-0.0012}^{+0.0003+0.0017}$ & $0.0021_{-0.0010-0.0013}^{+0.0004+0.0017}$ & $0.0011_{-0.0010-0.0013}^{+0.0004+0.0017}$ \\
\hline$\Omega_{m 0}$ & $0.3013_{-0.0097-0.0175}^{+0.0089+0.0182}$ & $0.3035_{-0.0098-0.0168}^{+0.0089+0.0184}$ & $0.3010_{-0.0089-0.0165}^{+0.0084+0.0165}$ \\
\hline$\chi_{\min }^{2}$ & 13721.704 & 13720.834 & 13719.138 \\
\hline
\end{tabular}




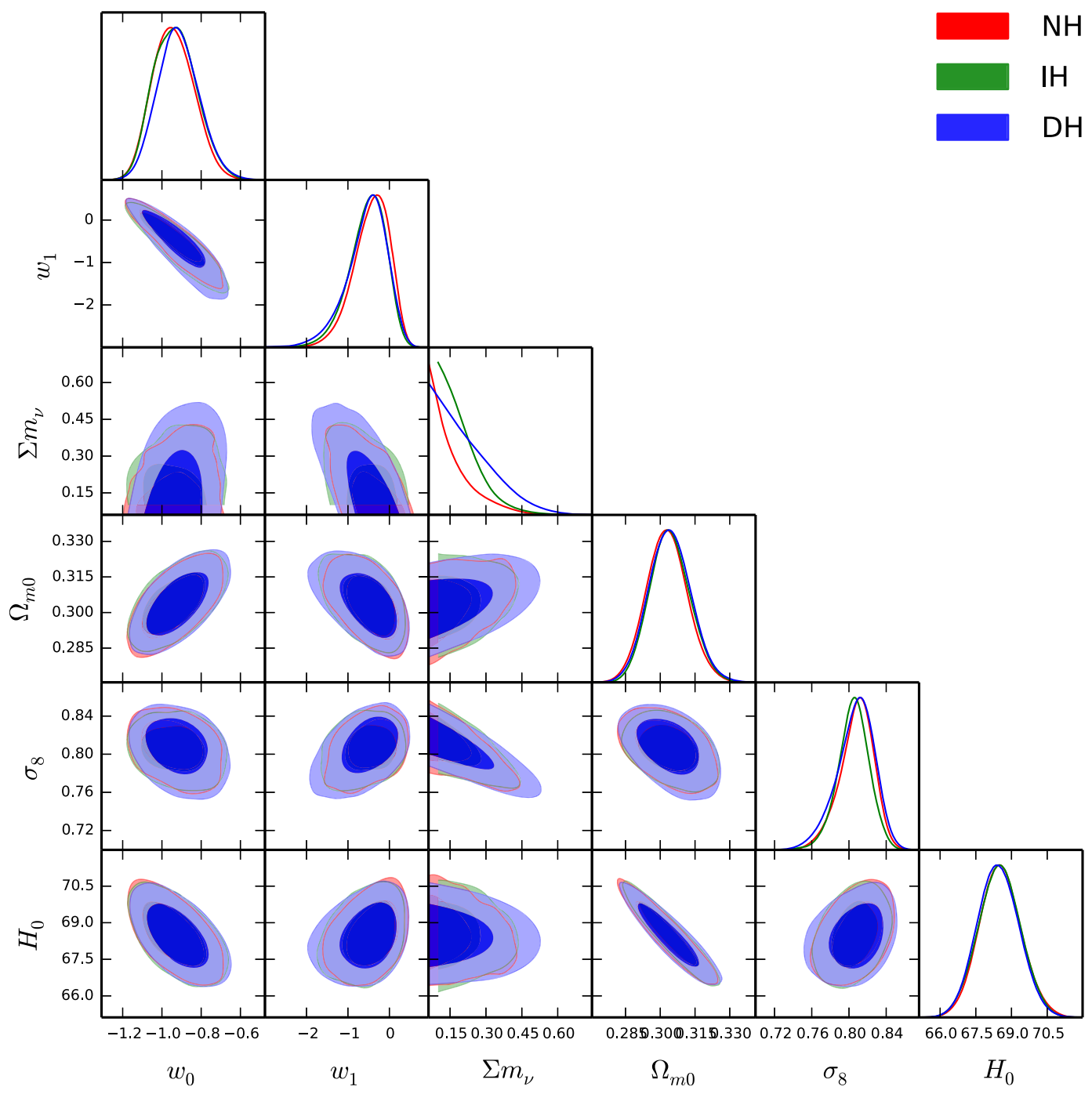

FIG. 1. $68 \%$ and $95 \%$ confidence-level contour plots for some selected parameters of the CPL parametrization considering three different neutrino mass hierarchies, namely the $\mathrm{NH}, \mathrm{IH}$, and $\mathrm{DH}$ using the $\mathrm{CMB}+\mathrm{SNIa}+\mathrm{BAO}+\mathrm{RSD}+\mathrm{WL}+\mathrm{CC}+H_{0}$ data set.

Sky Survey Data Release 7 (SDSS DR7) data [75], and finally $r_{s}\left(z_{d}\right) / D_{V}(z=0.57)=0.0732 \pm 0.0012$ from the SDSS DR9 [76].

(iv) Redshift space distortion (RSD): We use RSD data from different observational surveys from 2dFGRS [77], the WiggleZ [78], the SDSS LRG [79], the BOSS CMASS [80], the 6dFGRS [81], and the VIPERS [82]. The measured values of the RSD data can be found in Table I of Ref. [83].

(v) Weak lensing (WL) data: We consider the weak gravitational lensing data from the blue galaxy sample compiled from the Canada-France-Hawaii Telescope Lensing Survey (CFHTLenS) $[84,85]$ for our analysis.

(vi) Cosmic chronometers (CC) plus the local value of the Hubble parameter $\left(\mathrm{CC}+H_{0}\right)$ : We employ the recently released cosmic chronometer data comprising 30 measurements of the Hubble parameter in the redshift interval $0<z<2$ [86]. Additionally we use the local value of the Hubble parameter yielding
$H_{0}=73.02 \pm 1.79 \mathrm{~km} / \mathrm{s} / \mathrm{Mpc}$ with $2.4 \%$ precision as reported recently in [87].

We modified the publicly available CosmoMc code [88] to obtain the Markov chain Monte Carlo samples using uniform priors on the free parameters

$P=\left\{\omega_{b}, \omega_{d m}, 100 \theta_{M C}, \ln 10^{10} A_{s}, n_{s}, \tau_{\text {reio }}, \sum m_{\nu}, w_{0}, w_{1}\right\}$,

where $\omega_{b}=\Omega_{b} h^{2}, \omega_{d m}=\Omega_{d m} h^{2}$ are respectively the baryon density and the cold dark matter density; $\theta_{M C}$ is the approximation to the angular size of the sound horizon at last scattering; $A_{s}$ is defined to be the amplitude of the initial power spectrum; $n_{s}$ is the spectral index; $\tau_{\text {reio }}$ is the optical depth due to reionization; $\sum m_{\nu}$ is the total neutrino mass; and $w_{0}, w_{1}$ are the model parameters which have been defined previously. The priors used for the model parameters are $\omega_{b} \in[0.005,0.1] ; \omega_{m} \in[0.01,0.99]$; $100 \theta_{M C} \in[0.5,10] ; \ln \left(10^{10} A_{s}\right) \in[2.4,4] ; n_{s} \in[0.5,1.5] ;$ 
$\tau_{\text {reio }} \in[0.01,0.8] ; w_{0} \in[-2,0]$ and $w_{1} \in[-3,3]$ for the CPL and JBP models while for the logarithmic parametrization $w_{0} \in[-2,0]$ and $w_{1} \in[-3,0]$. In order to constrain the free parameters of the models, we consider three dynamical $\mathrm{DE}$ models in the $\mathrm{NH}, \mathrm{IH}$, and $\mathrm{DH}$ with minimum sums of the neutrino mass to be $0.06 \mathrm{eV}, 0.1 \mathrm{eV}$, and $0.0 \mathrm{eV}$, respectively, for the three species of active neutrinos. In what follows, for the present analysis, we have considered the parametrized post-Friedmann (PPF) approximation $[89,90]$ for three DE models. In particular, for the logarithmic model in Eq. (7) if the prior on $w_{1}$ is considered to be positive, i.e. $w_{1}>0$, then when the function $w(z)$ is evaluated at very early times, it has a positive divergence, implying $w_{d e}>-1 / 3$ in the radiation era. Therefore, to avoid such problems, we have fixed the prior as $w_{1} \leq 0$ during the statistical analysis. For all dynamical DE models we run the Monte Carlo Markov chains until the parameters converge to a parameter according to the Gelman-Rubin criteria $R-1<0.01$ [91].

\section{RESULTS OF THE ANALYSIS}

Let us summarize the main observational results extracted from the dynamical dark energy models in the presence of massive neutrinos at three different hierarchies, namely the $\mathrm{NH}, \mathrm{IH}$, and $\mathrm{DH}$, using the combined observational data, $\mathrm{CMB}+\mathrm{SNIa}+\mathrm{BAO}+\mathrm{RSD}+\mathrm{WL}+\mathrm{CC}+H_{0}$, described in Sec. III. In Tables I, II, and III we summarize the main results of the statistical analysis for the CPL, logarithmic, and JBP models, respectively.

Figure 1 shows the one-dimensional marginalized distribution and $68 \%$, 95\% C.L. regions for some selected parameters of the CPL model. We note that $\sum m_{\nu}<$ $0.353,0.368,0.444 \mathrm{eV}$ at $95 \%$ C.L., for $\mathrm{NH}$, IH and $\mathrm{DH}$, respectively. In general, our analysis reveals that all three neutrino hierarchies return similar constraints on the baseline parameters. However, a slight variational effect depending on different hierarchies of the neutrino masses is observed on the DE parameter $w_{1}$. In particular we observe

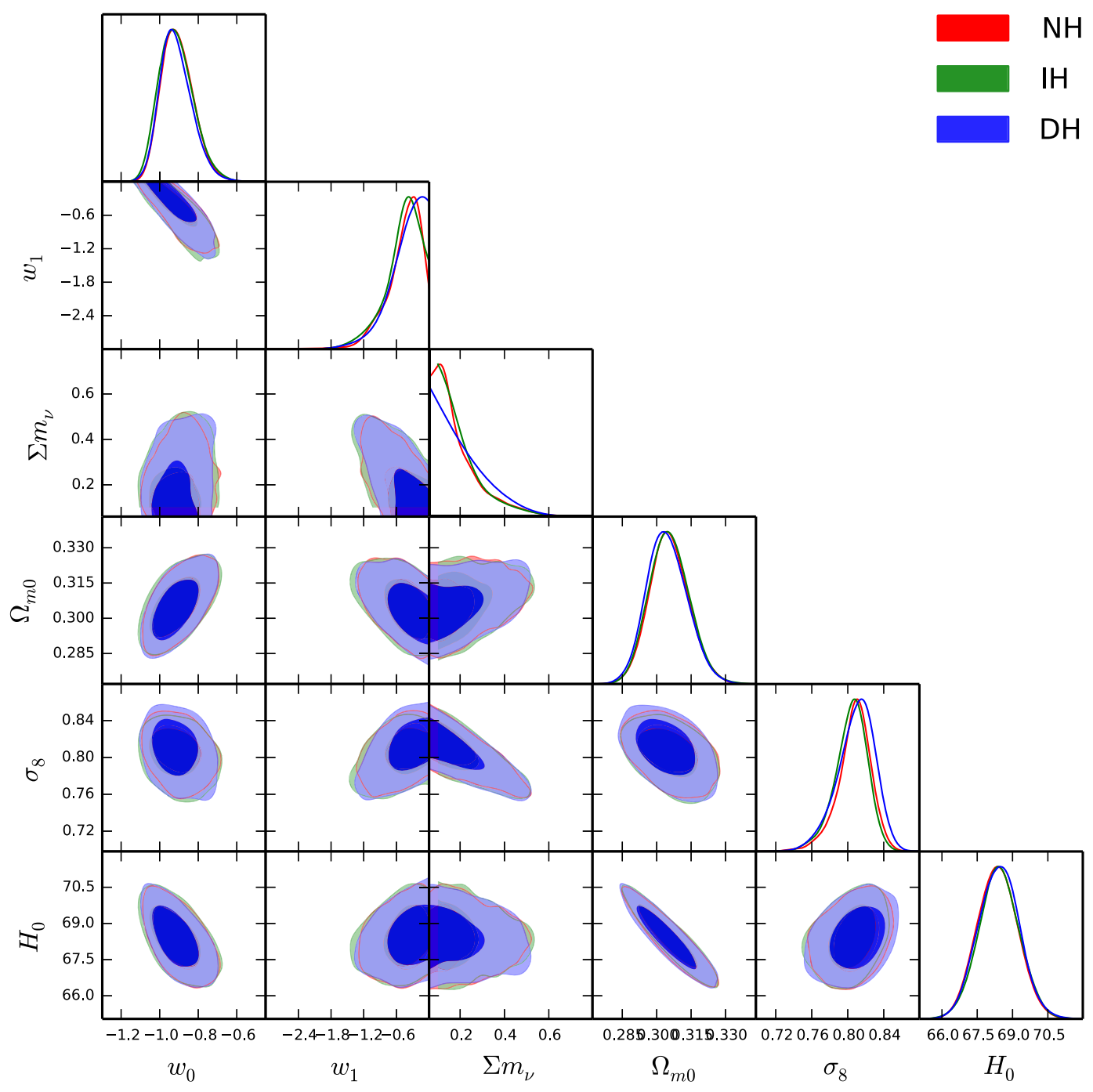

FIG. 2. $68 \%$ and $95 \%$ confidence-level contour plots for some selected parameters of the logarithmic parametrization considering three different neutrino mass hierarchies, namely the $\mathrm{NH}, \mathrm{IH}, \mathrm{DH}$ using $\mathrm{CMB}+\mathrm{SNIa}+\mathrm{BAO}+\mathrm{RSD}+\mathrm{WL}+\mathrm{CC}+H_{0}$. 


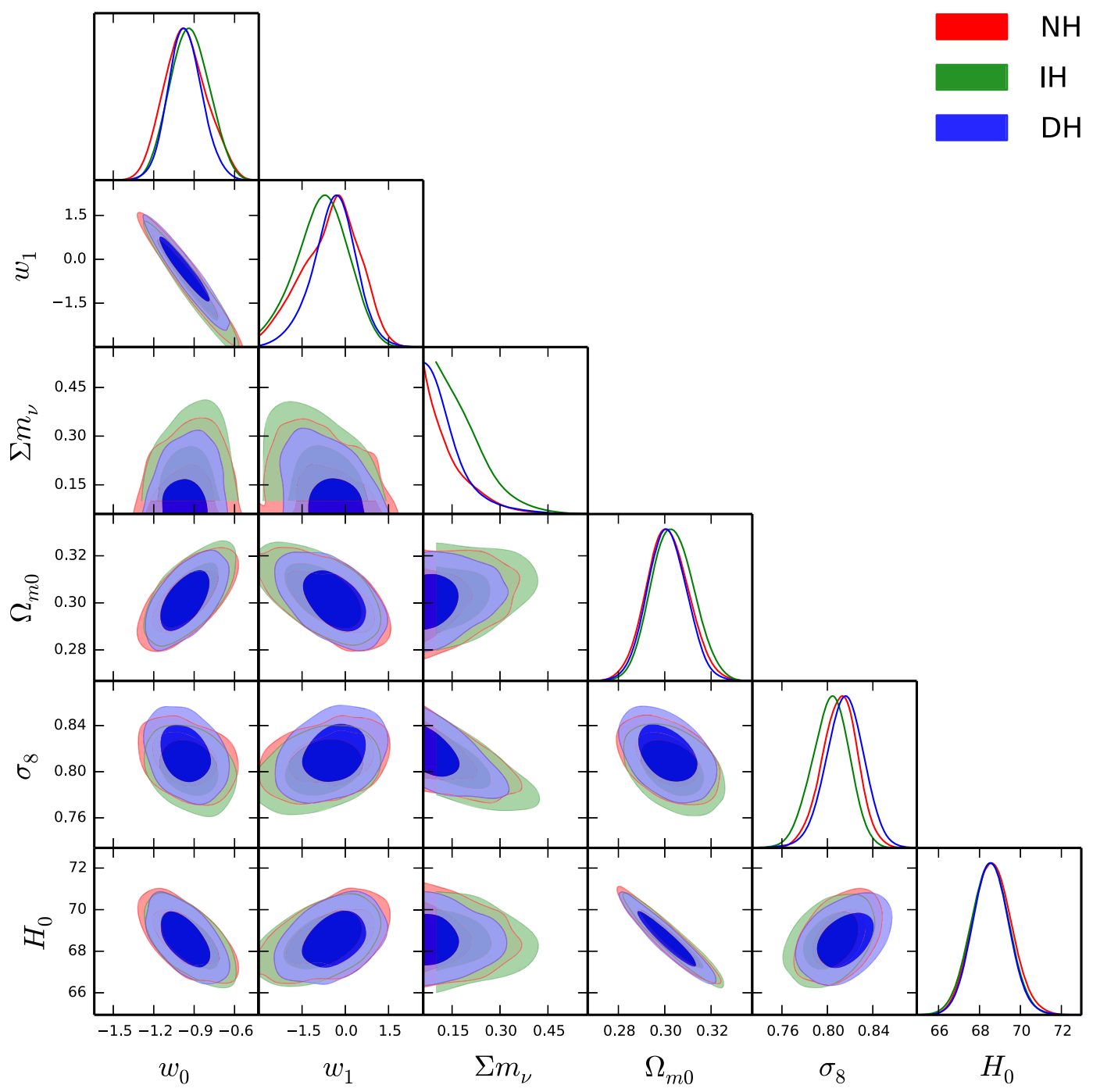

FIG. 3. $68 \%$ and $95 \%$ confidence-level contour plots for some selected parameters of the JBP model considering three different neutrino mass hierarchies, namely the $\mathrm{NH}, \mathrm{IH}$, DH using $\mathrm{CMB}+\mathrm{SNIa}+\mathrm{BAO}+\mathrm{RSD}+\mathrm{WL}+\mathrm{CC}+H_{0}$.

that the maximum variation of $w_{1}$ (from its best-fit values) ${ }^{1}$ is of order $\Delta w_{1} \sim 0.1$.

Figures 2 and 3 show the one-dimensional marginalized distribution and the parametric space at $68 \%, 95 \%$ C.L. regions for some selected parameters of the logarithmic and JBP models, respectively. We can note that no significant variations are observed in the full parameter base of the logarithmic parametrization, including DE properties $\left(w_{0}\right.$ and $\left.w_{1}\right)$. Within the logarithmic model we note that $\sum m_{\nu}<$ $0.412,0.428,0.425 \mathrm{eV}$ at $95 \%$ C.L., for $\mathrm{NH}, \mathrm{IH}$ and $\mathrm{DH}$, respectively. On the other hand, in the JBP model we note the significant variations on the DE parameter. In particular, on $w_{1}$, we find that $\Delta w_{1}=0.2883$ (or -0.1116 ) when $\mathrm{NH}$ is

\footnotetext{
${ }^{1}$ Here we define the variation as $\Delta w_{1}=w_{1}$ (at $\left.\mathrm{NH}\right)-w_{1}$ (at $\mathrm{IH})\left[\right.$ or $w_{1}($ at $\left.\mathrm{DH})\right]$.
}

compared to IH or DH. Here, we note $\sum m_{\nu}<0.294,0.348$, $0.253 \mathrm{eV}$ at $95 \%$ C.L., for $\mathrm{NH}, \mathrm{IH}$ and $\mathrm{DH}$, respectively.

In general, taking into account the neutrino mass splittings, for instance within the CPL model with the $\mathrm{NH}$ scheme, that works out to be two neutrinos of approximately $0.10 \mathrm{eV}$ and one slightly heavier neutrino of $0.15 \mathrm{eV}$. This scenario is almost degenerate. The other hierarchy schemes present practically the same upper limits on the neutrino mass splittings; therefore, we really should expect nearly identical results on the baseline of the model. The same interpretation applies to both logarithmic and JBP models. Additionally, in Fig. 4 we show the theoretical predictions of the angular CMB power spectrum temperature anisotropy for the three dynamical DE models considered in this work in comparison to the $\Lambda \mathrm{CDM}$ model. In those plots we have assumed the best-fit values from the tables for each respective model. Evidently one can clearly observe that significant variations are not 

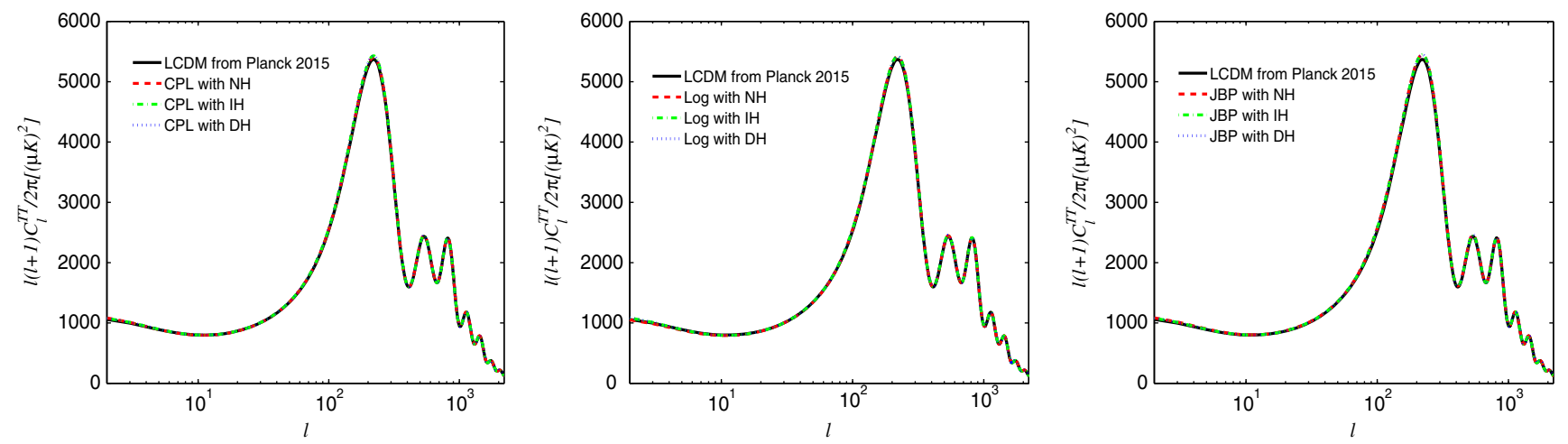

FIG. 4. The CMB TT power spectrum for CPL (left panel), logarithmic (middle panel), and JBP (right panel) models for the three different mass hierarchy schemes, compared to the $\Lambda$ CDM TT power spectrum. We note that in all three plots the spectra for different hierarchies as well as for $\Lambda \mathrm{CDM}$ are completely indistinguishable from one another.
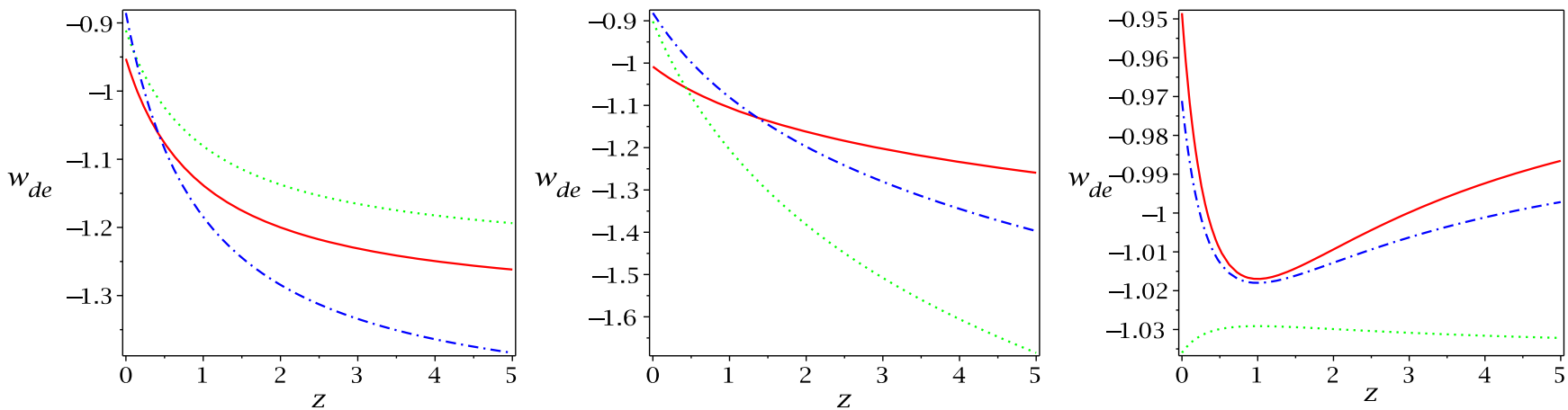

FIG. 5. Evolution of the DE EoS as a function of the redshift for CPL (left panel), logarithmic (middle panel) and JBP (right panel) parametrizations at three different neutrino mass hierarchies have been shown for the best-fit values of the model parameters $w_{0}$ and $w_{1}$ using the combined analysis $\mathrm{CMB}+\mathrm{SNIa}+\mathrm{BAO}+\mathrm{RSD}+\mathrm{WL}+\mathrm{CC}+H_{0}$. In each plot, the solid, dotted and dashed-dotted curves stand for $\mathrm{NH}, \mathrm{IH}$ and $\mathrm{DH}$, respectively.

observed in the behavior of the dynamical DE models when the presence of massive neutrinos are taken into account in the cosmological picture. In fact, our constraints are very close to the $\Lambda \mathrm{CDM}$ cosmology.

Figure 5 shows the quantitative evolution of the DE EoS considering three distinct neutrino mass schemes for the CPL, logarithmic and JBP models. From the figure, we see that at high redshifts, the EoS for the DE parametrizations exhibit significant deviations at three different hierarchies. However, we notice that for $z \sim 0$, the EoS at different neutrino mass hierarchies for the CPL and logarithmic models become close to each other, while in the JBP parametrization, the EoS curves for $\mathrm{NH}$ and $\mathrm{DH}$ are similar in contrast to the EoS curve at $\mathrm{IH}$.

\section{FINAL REMARKS}

The presence of massive neutrinos is an essential piece in the dynamics of the Universe, and it is known that their properties can correlate in different ways with other cosmological parameters. Thus, the determination of its properties with accurate and robust methods plays an important role in a particular cosmological model.

In this work we have measured the effects of massive neutrinos via three different neutrino hierarchies, namely $\mathrm{NH}, \mathrm{IH}$, and $\mathrm{DH}$, on the cosmological scenarios where $\mathrm{DE}$ offers a dynamical character. We consider three well-known and most-used dynamical DE models represented by the CPL, logarithmic and JBP parametrizations. The models have been constrained using the most current observational data from CMB, SNIa, BAO, RSD, WL, CC, and $H_{0}$. From

TABLE IV. Summary of the cosmological neutrino mass bound $\left(\sum m_{\nu}\right)$ at $95 \%$ C.L., for the three dynamical DE models considered here. The mass is in the units of $\mathrm{eV}$.

\begin{tabular}{|c|c|c|c|}
\hline Models & $\mathrm{NH}$ & $\mathrm{IH}$ & DH \\
\hline CPL model & $\sum m_{\nu}<0.353$ & $\sum m_{\nu}<0.368$ & $\sum m_{\nu}<0.42$ \\
\hline $\begin{array}{l}\text { Logarithmic } \\
\text { model }\end{array}$ & $\sum m_{\nu}<0.412$ & $<0.428$ & 0.425 \\
\hline JBP model & $\sum m_{\nu}<0.294$ & $\sum m_{\nu}<0.348$ & $\sum m_{\nu}<0.253$ \\
\hline
\end{tabular}


the combined analysis of these observational data, we provide with robustness a cosmological neutrino mass bound (see Table IV) in the presence of the dynamical DE models. Further, we have found that the fixation of different neutrino mass hierarchies does not exhibit any significant variation on the baseline of parameters of the models, except on the DE parameter $w_{1}$ in the CPL and JBP parameterizations. But, such variations do not present statistical deviations from the $\Lambda \mathrm{CDM}$ model. In general, we can summarize our results by concluding that independent of the dynamic nature of DE, different choices of neutrino mass scheme throughout the cosmic history will not make significant changes to the dynamic properties of DE within each model.

\section{ACKNOWLEDGMENTS}

The authors thank the anonymous referee for his/her detailed comments and suggestions which improved the work significantly. Also, the authors are grateful to Thomas Tram, Lixin $\mathrm{Xu}$, and Vinicius Miranda for helpful discussions. W. Yang's work is supported by the National Natural Science Foundation of China under Grant No. 11647153, the Foundation of Education Department of Liaoning Province in China under Grant No. L201683666, and the Youth Foundation of Liaoning Normal University under Grant No. LS2015L003. S. P. is supported by the SERB-NPDF grant (Grant No. PDF/2015/000640), Government of India. D. F. M. acknowledges the support from the Research Council of Norway.
[1] M. C. G. Garcia and M. Maltoni, Phys. Rep. 460, 1 (2008).

[2] T. Araki et al. (KamLAND Collaboration), Phys. Rev. Lett. 94, 081801 (2005); S. Abe et al. (KamLAND Collaboration), Phys. Rev. Lett. 100, 221803 (2008).

[3] Y. Ashie et al. (Super-Kamiokande Collaboration), Phys. Rev. D 71, 112005 (2005).

[4] K. A. Olive et al., Chin. Phys. C 38, 090001 (2014).

[5] A. de Gouvea et al. (Intensity Frontier Neutrino Working Group Collaboration), arXiv:1310.4340.

[6] X. Qian and P. Vogel, Prog. Part. Nucl. Phys. 83, 1 (2015).

[7] F. De Bernardis, T. D. Kitching, A. Heavens, and A. Melchiorri, Phys. Rev. D 80, 123509 (2009).

[8] Q. G. Huang, K. Wang, and S. Wang, Eur. Phys. J. C 76, 489 (2016).

[9] L. Xu and Q. G. Huang, arXiv:1611.05178.

[10] R. Jimenez, T. Kitching, C. P. Garay, and L. Verde, J. Cosmol. Astropart. Phys. 05 (2010) 035.

[11] M. Gerbino, M. Lattanzi, O. Mena, and K. Freese, arXiv:1611.07847.

[12] S. Vagnozzi et al., arXiv:1701.08172.

[13] A. D. Dolgov, Phys. Rep. 370, 333 (2002).

[14] J. Lesgourgues and S. Pastor, Phys. Rep. 429, 307 (2006).

[15] P. A. R. Ade et al. (Planck Collaboration), Astron. Astrophys. 594, A13 (2016).

[16] B. Audren et al., J. Cosmol. Astropart. Phys. 03 (2015) 036.

[17] E. D. Valentino et al., arXiv:1612.00021.

[18] C. Q. Geng, C. C. Lee, and J. L. Shen, Phys. Lett. B 740, 285 (2015).

[19] J. Lu, M. Liu, Y. Wu, Y. Wang, and W. Yang, Eur. Phys. J. C 76, 679 (2016).

[20] J. F. Zhang, M. M. Zhao, Y. H. Li, and X. Zhang, J. Cosmol. Astropart. Phys. 04 (2015) 038

[21] S. Wang, Y. F. Wang, D. M. Xia, and X. Zhang, Phys. Rev. D 94, 083519 (2016).

[22] Y. Chen and L. Xu, Phys. Lett. B 752, 66 (2016).

[23] C. Q. Geng, C. C. Lee, R. Myrzakulov, M. Sami, and E. N. Saridakis, J. Cosmol. Astropart. Phys. 01 (2016) 049.
[24] Y. Chen, B. Ratra, M. Biesiada, S. Li, and Z. H. Zhu, Astrophys. J. 829, 61 (2016).

[25] S. Kumar and R. C. Nunes, Phys. Rev. D 94, 123511 (2016).

[26] S. Kumar and R. C. Nunes, arXiv:1702.02143.

[27] R. Y. Guo, Y.H. Li, J.F. Zhang, and X. Zhang, arXiv: 1702.04189.

[28] J. L. Bernal, L. Verde, and A. G. Riess, J. Cosmol. Astropart. Phys. 10 (2016) 019.

[29] E. Di Valentino, A. Melchiorri, and J. Silk, Phys. Lett. B 761, 242 (2016).

[30] M. Archidiacono, S. Gariazzo, C. Giunti, S. Hannestad, R. Hansen, M. Laveder, and T. Tram, J. Cosmol. Astropart. Phys. 08 (2016) 067.

[31] M. Gerbino, K. Freese, S. Vagnozzi, M. Lattanzi, O. Mena, E. Giusarma, and S. Ho, Phys. Rev. D 95, 043512 (2017).

[32] T. Tram, R. Vallance, and V. Vennin, J. Cosmol. Astropart. Phys. 01 (2017) 046.

[33] E. D. Valentino and F. R. Bouchet, J. Cosmol. Astropart. Phys. 10 (2016) 011.

[34] E. J. Copeland, M. Sami, and S. Tsujikawa, Int. J. Mod. Phys. D 15, 1753 (2006).

[35] L. Amendola, Phys. Rev. D 62, 043511 (2000).

[36] T. Koivisto and D. F. Mota, Phys. Lett. B 644, 104 (2007).

[37] D. F. Mota, J. R. Kristiansen, T. Koivisto, and N.E. Groeneboom, Mon. Not. R. Astron. Soc. 382, 793 (2007).

[38] R. Gannouji, B. Moraes, D. F. Mota, D. Polarski, S. Tsujikawa, and H. A. Winther, Phys. Rev. D 82, 124006 (2010).

[39] D. F. Mota, D. J. Shaw, and J. Silk, Astrophys. J. 675, 29 (2008).

[40] C. Llinares, D. F. Mota, and H. A. Winther, Astron. Astrophys. 562, A78 (2014).

[41] C. Llinares and D. Mota, Phys. Rev. Lett. 110, 161101 (2013).

[42] D. F. Mota, M. Sandstad, and T. Zlosnik, J. High Energy Phys. 12 (2010) 051.

[43] L. Xu, Phys. Rev. D 87, 043525 (2013).

[44] L. Xu, Phys. Rev. D 85, 123505 (2012). 
[45] R. C. Nunes and D. Pavón, Phys. Rev. D 91, 063526 (2015).

[46] R. C. Nunes and S. Pan, Mon. Not. R. Astron. Soc. 459, 673 (2016).

[47] S. Pan, J. de Haro, A. Paliathanasis, and R. J. Slagter, Mon. Not. R. Astron. Soc. 460, 1445 (2016).

[48] J. de Haro, J. Amorós, and S. Pan, Phys. Rev. D 93, 084018 (2016).

[49] J. de Haro, J. Amorós, and S. Pan, Phys. Rev. D 94, 064060 (2016).

[50] W. Yang, L. Xu, Y. Wang, and Y. Wu, Phys. Rev. D 89, 043511 (2014).

[51] R. C. Nunes, E. M. Barboza, Jr., E. M. C. Abreu, and J. A. Neto, J. Cosmol. Astropart. Phys. 08 (2016) 051.

[52] W. Yang and L. Xu, Phys. Rev. D 89, 083517 (2014).

[53] W. Yang and L. Xu, J. Cosmol. Astropart. Phys. 08 (2014) 034.

[54] R. C. Nunes and E. M. Barboza, Gen. Relativ. Gravit. 46, 1820 (2014).

[55] S. Pan, S. Bhattacharya, and S. Chakraborty, Mon. Not. R. Astron. Soc. 452, 3038 (2015).

[56] R. C. Nunes, S. Pan, and E. N. Saridakis, Phys. Rev. D 94, 023508 (2016).

[57] W. Yang, H. Li, Y. Wu, and J. Lu, J. Cosmol. Astropart. Phys. 10 (2016) 007.

[58] G. Pantazis, S. Nesseris, and L. Perivolaropoulos, Phys. Rev. D 93, 103503 (2016).

[59] G. S. Sharov, S. Bhattacharya, S. Pan, R. C. Nunes, and S. Chakraborty, Mon. Not. R. Astron. Soc. 466, 3497 (2017).

[60] M. Moresco, R. Jimenez, L. Verde, A. Cimatti, L. Pozzetti, C. Maraston, and D. Thomas, J. Cosmol. Astropart. Phys. 12 (2016) 039.

[61] C. E. Rivera, Galaxies 4, 8 (2016).

[62] Y. Y. Xu and X. Zhang, Eur. Phys. J. C 76, 588 (2016).

[63] P. A. R. Ade et al. (Planck Collaboration), Astron. Astrophys. 594, A14 (2016).

[64] M. Chevallier and D. Polarski, Int. J. Mod. Phys. D 10, 213 (2001).

[65] E. V. Linder, Phys. Rev. Lett. 90, 091301 (2003).

[66] G. Efstathiou, Mon. Not. R. Astron. Soc. 310, 842 (1999).

[67] H. K. Jassal, J. S. Bagla, and T. Padmanabhan, Mon. Not. R. Astron. Soc. 356, L11 (2005).

[68] V. F. Mukhanov, H. A. Feldman, and R. H. Brandenberger, Phys. Rep. 215, 203 (1992).

[69] C. P. Ma and E. Bertschinger, Astrophys. J. 455, 7 (1995).
[70] K. A. Malik and D. Wands, Phys. Rep. 475, 1 (2009).

[71] R. Adam, P. A. R. Ade, N. Aghanim et al. (Planck Collaboration), Astron. Astrophys. 594, A1 (2016).

[72] N. Aghanim, M. Arnaud, M. Ashdown et al. (Planck Collaboration), Astron. Astrophys. 594, A11 (2016).

[73] M. Betoule et al. (SDSS Collaboration), Astron. Astrophys. 568, A22 (2014).

[74] F. Beutler, C. Blake, M. Colless, D. H. Jones, L. StaveleySmith, L. Campbell, Q. Parker, W. Saunders, and F Watson, Mon. Not. R. Astron. Soc. 416, 3017 (2011).

[75] N. Padmanabhan, X. Xu, D. J. Eisenstein, R. Scalzo, A. J. Cuesta, K. T. Mehta, and E. Kazin, Mon. Not. R. Astron. Soc. 427, 2132 (2012).

[76] M. Manera et al., Mon. Not. R. Astron. Soc. 428, 1036 (2013).

[77] W. J. Percival et al. (2dFGRS Collaboration), Mon. Not. R. Astron. Soc. 353, 1201 (2004).

[78] C. Blake et al., Mon. Not. R. Astron. Soc. 415, 2876 (2011).

[79] L. Samushia, W. J. Percival, and A. Raccanelli, Mon. Not. R. Astron. Soc. 420, 2102 (2012).

[80] B. A. Reid et al., Mon. Not. R. Astron. Soc. 426, 2719 (2012).

[81] F. Beutler, C. Blake, M. Colless, D. H. Jones, L. StaveleySmith, G. B. Poole, L. Campbell, Q. Parker, W. Saunders, and F. Watson, Mon. Not. R. Astron. Soc. 423, 3430 (2012).

[82] S. de la Torre et al., Astron. Astrophys. 557, A54 (2013).

[83] W. Yang and L. Xu, Phys. Rev. D 90, 083532 (2014).

[84] C. Heymans et al., Mon. Not. R. Astron. Soc. 432, 2433 (2013).

[85] M. Asgari, C. Heymans, C. Blake, J. Harnois-Deraps, P. Schneider, and L. Van Waerbeke, Mon. Not. R. Astron. Soc. 464, 1676 (2017).

[86] M. Moresco, L. Pozzetti, A. Cimatti, R. Jimenez, C. Maraston, L. Verde, D. Thomas, A. Citro, R. Tojeiro, and D. Wilkinson, J. Cosmol. Astropart. Phys. 05 (2016) 014.

[87] A. G. Riess et al., Astrophys. J. 826, 56 (2016).

[88] A. Lewis and S. Bridle, Phys. Rev. D 66, 103511 (2002); http://cosmologist.info/cosmomc/.

[89] W. Fang, W. Hu, and A. Lewis, Phys. Rev. D 78, 087303 (2008).

[90] W. Hu, Phys. Rev. D 77, 103524 (2008).

[91] A. Gelman and D. Rubin, Stat. Sci. 7, 457 (1992). 